from one group little energy transfer occurs to the pigment absorbing at $890 \mathrm{~m} \mu$ whilst from the alternate group transfer occurs with high efficiency. The problem of energy transfer between different pigment systems is discussed by Pearlstein and by Robinson, who consider the movement of localized excitons as a pcssible mechan. ism.

In a second section, the papers are mainly concerned with observations on the change in absorption spectrum which occurs during photosynthesis. It is generally believed that two successive photochemical steps, separated by a thermochemical reaction, are involved in the production of the ultimate photochemical reductant. Certain probable intermediates, cytochromes for example, change their absorption spectrum on oxidation and reduction and part of the absorption change observed in photosynthesis may be related to the reversible oxidation and reduction of eytochromes. In an introductory paper, Chance et al. summarize what is known about the kinetics of the changes and discuss how far it is possible to assign definitively individual cytochrome components to particular sites in the electron transport chain. A number of workers, using the double beam difference spectrophotometer developed by Chance, report observations on a variety of organisms: Nishimura on red algae, with particular reference to the effect of various photosynthetic inhibitors; Levine et al. with mutants of Chlamy. domonas; Avron and Chance, and independently Hind and Olson, with isolated spinach chloroplasts. From this wealth of data, the complexity of the absorption changes becomes apparent. Attempts have been made to separate from chloroplasts two types of particles in which the two photoreactions are present to different extents. Vernon et al. discuss further studies on particles obtained by fractionation of chloroplasts in the presence of detergents or after sonication. They have separated heavier particles containing relatively more of a photo-system activated by shorter wavelengths and lighter particles which contain relatively more of a photosystem activated by longer wavelengths.

In the third section, the biochemical analysis of the catalytic components involved is considered and in particular the relationship between light induced electron transport and the formation of energy rich phosphate compounds. The "chemi-osmotic" hypothesis is discussed by a number of contributors and is, at the present time, guiding many investigations. McCarty and Racker report the separation of a "coupling" factor which links the formation of some high energy state in the chloroplast to its subsequent utilization in the formation of phosphate esters. Jagendorf and Uribe write at length on the general evidence that phosphorylation in chloroplasts is related to the formation of a proton gradient and, in particular, give experimental evidence that phosphorylation can be stimulated in the dark in chloroplasts as a result of transferring them from an acid to an alkaline medium. Dilley reports evidence concerning the permeability properties of chloroplasts and considers that chloroplasts consist of an "inner" and an "outer" space. This "inner" compartment shrinks and swells in response to changes of osmotic pressure and $p \mathrm{H}$. Measurements of the marked change in volume exhibited by chloroplasts as a consequence of illumination were reported by Packer, Deamer and Crofts, using either a Coulter counter or the change in intensity of light scattering.

The section on the fine structure of chloroplasts includes contributions by Menke and by Park and Branton, who discuss the nature of the thylakoid membrane and the location of the quantasomes within them. Weier, Stocking and Shumway discuss the organization of the plastid at the molecular level and in particular the position and orientation of the pigment molecules.

This collection of papers will be of great value to the research worker, bringing together observations based on different scientific disciplines. It is too specialized for the general reader and rather provides a cross section of current activity in the field, than presenting a synthesis of the most significant information.

C. P. Whittingham

\section{ALL ASPECTS OF SEAWEED}

\section{Proceedings of the Fifth International Seaweed Sympo-} sium, Halifax, Canada

August 25-28, 1965. Edited by E. Gordon Young and J. L. MeLachlan. Pp. xviii +424 . (Oxford, London and New York: Pergamon Press, Ltd., 1966.) 100s. net.

The proceedings of this symposium, held in Halifax, Nova Scotia, in August 1965, include an extraordinary variety of communications varying in length from substantial reviews to brief abstracts of work published in full elsewhere. Among much of interest in this miscellany only a small sample can be mentioned. Although some of the ecological contributions are largely descriptive, as, for example, two which deal with the varied and abundant Antarctic seaweed flora, others deal with more dynamic aspects. Of particular interest is the investigation by workers, both in California and the Isle of Man, of the devastating effects of browsing sea-urchins on kelp beds. Studies by Drs Conover and Sieburth of the effects of tannins excreted by Phaeophyceae on epiphytes and planktonic animals suggest that such substances may be of great importance in inshore waters in determining distribution and succession. Of the many papers dealing with purely chemical topics the review by Professor Araki of the structural chemistry of agar polysaccharides will be welcomed as an authoritative account of a difficult subject. Both biologists and chemists will be intrigued by the report of workers at the Atlantic Regional Laboratory at Halifax of pure crystalline fibres of chitan as an extracellular product of the diatom, Thalassiosira fluviatilis.

In view of the unique way in which the International Seaweed Symposia bring together academic scientists and industrialists, it is disappointing that the seaweed industry has not developed more quickly. Dr Woodward makes a brave attempt to predict what the future of the industry may be. After reading the papers on the uses of seaweed products I am inclined to share his optimism, for the possibilities are both varied and promising. Seaweed products find use on the one hand in medicine, for example, both in the treatment of tumours and as tablet disintegrants, and, on the other, in civil engineering, in controlling water flow in porous ground. Some of the effects of seaweed extracts on the growth of plants and the reduction of disease and pest infestation savour a little of "muck and magic" but seem definite and well worth further investigation.

G. E. FogG

\section{PAPERBACK PALAEONTOLOGY}

\section{General Palaeontology}

By A. Brouwer. Translated by R. H. Kaye. Pp. viii +216 . (Edinburgh and London: Oliver and Boyd, Ltd., 1967.) $35 s$.

THIs volume of five chapters is a translation of Brouwer's Adgemene Paleontologie first published in 1959. A few more recent references have been added, but almost no textural change has been made. The translation has been very well done and the prose is most readable. The preface states that this is the first textbook covering the whole field of general palaeontology since Walther's Allgemeine Paläontologie and there can be no doubt that the collation of information is most useful even if much of it can already be found in the standard textbooks by Simpson, Ager, Rhodes and others. This textbook has the ad- 